\title{
Interpregnancy interval, maternal age, and offspring's BMI and blood pressure at 7 years of age
}

\author{
Shenghui $\mathrm{Li}^{1,2} \cdot$ Jin $\mathrm{Hua}^{3} \cdot$ Haifa Hong ${ }^{4}$ Yanling Wang ${ }^{5}$ Jun Zhang ${ }^{1,2}$
}

Received: 25 July 2017 / Revised: 27 September 2017 / Accepted: 20 October 2017 / Published online: 23 February 2018

(c) The Author(s) 2018. This article is published with open access

\begin{abstract}
Interpregnancy interval and maternal age are associated with birth outcomes. However, it is unknown regarding their long-term effects on child health. We aim to assess the associations between interpregnancy interval and offspring's body mass index (BMI) and blood pressure (BP) at age of 7 years and to examine the role of maternal age in the associations. A secondary analysis was performed among 2604 mother-infant pairs in the prospective National Collaborative Perinatal Project, in which the children were followed up until $7 \mathrm{yrs}$ of age. Interpregnancy interval was positively associated with offspring's diastolic BP at 7 yrs $(\beta=0.053,95 \% \mathrm{CI}: 0.004-0.102)$ after adjusting for maternal and perinatal characteristics, feeding pattern, rapid weight gain in the first year of life, and current BMI $\mathrm{z}$ score and height $\mathrm{z}$ score. The inclusion of maternal age in the model did not change the effect size. Maternal age was independently associated with offspring's BMI z score at 7 yrs $(\beta=0.014,95 \%$ CI: 0.001-0.027). An interaction between interpregnancy interval and maternal age was present in the association with diastolic BP $(P=0.019)$, and the increasing maternal age aggravated the effects of long interpregnancy interval. Our finding suggests long interpregnancy interval is a risk factor for higher diastolic BP of the offspring. Increasing maternal age could amplify the impact. Our study challenges the current WHO recommendation for ideal interpregnancy interval, and we would suggest lowering the recommendation to $<24$ months and even shorter for women of advanced age.
\end{abstract}

Electronic supplementary material The online version of this article (https://doi.org/10.1038/s41371-018-0035-4) contains supplementary material, which is available to authorized users.

Shenghui Li

1sh9907@163.com

$\triangle$ Jun Zhang

zhangjun@xinhuamed.com.cn

1 MOE-Shanghai Key Laboratory of Children's Environmental Health, Xinhua Hospital, School of Medicine, Shanghai Jiaotong University, Shanghai, China

2 School of Public Health, Shanghai Jiaotong University, Shanghai, China

3 Shanghai First Maternity and Infant Hospital Corporation, Shanghai Tongji University, Shanghai, China

4 Shanghai Children's Medical Center, Shangai Jiaotong University School of Medicine, Shanghai, China

5 International Peace Maternity and Child Health, Shanghai Jiaotong University, Shanghai, China

\section{Introduction}

Since early 1920 s, researchers have begun to investigate the impacts of interpregnancy interval, an interval between the birth of one child and the conception of the next, on perinatal outcomes [1]. Previous studies have linked short interpregnancy interval, $<6$ months, with adverse birth outcomes, including preterm birth [2, 3], low birth weight [2, 3], small size for gestational age [2, 4], congenital anomalies [5, 6], and infant mortality [2, 7]. Postpartum deficiencies in essential micronutrients and hormone imbalance were proposed to explain the associations [8,9]. There is growing evidence that fetal and early life events have not only short term effects on fetal growth and perinatal outcome but also long-term impacts on individual's health and disease susceptibility in later life $[10,11]$. In contrast to the association between interpregnancy interval and perinatal outcomes, much less is known about the role of interpregnancy interval in childhood growth and development.

A recent study indicated a possible interactive effects of interpregnancy interval and maternal age on perinatal health outcomes [12]. During the past decades, there is a growing trend of delaying childbirth to later reproductive years all 
around the world, especially in higher-income countries $[13,14]$. Previous studies have confirmed that maternal age is an important influential factor for both fetal and postnatal growth and development $[14,15]$. Therefore, maternal age should be given full consideration when examining the impacts of interpregnancy interval on perinatal outcomes and childhood development. The current WHO recommendation for ideal interpregnancy interval is not less than 24 months after a live birth [16]. However, recent studies are challenging the recommendation [17-19]. For example, two studies suggested that interpregnancy interval of 12-24 months seems to have the lowest risk of adverse birth outcomes [18, 19]. One study further suggested lowering the recommendation to only 18 months and even shorter for women of advanced age against the background of increasing maternal age of childbearing [18]. In view of the debate on appropriate interpregnancy interval, this study was designed to explore the long-term impacts of interpregnancy interval on childhood growth and development. Specifically, we aimed to address two issues: (1) are there associations of interpregnancy interval with body mass index (BMI) and blood pressure (BP) at age of 7 years? (2) is maternal age involved in the associations and what's the role of maternal age in this associations?

\section{Methods}

\section{Study subjects}

Participants in this study were selected from the Collaborative Perinatal Project (CPP). Detailed information on the CPP is available elsewhere [20]. Briefly, the CPP was a longitudinal cohort study that recruited 46,021 pregnant women with 56,990 pregnancies from 12 clinical sites in the US through 1959-1965. The women who came to these hospitals for prenatal care were invited to participate in the study, and their children were followed up until 7 years old.

This study focuses on pregnant women with two live singleton births. Among the 46,021 women, 2705 had two live singleton births during the enrollment period. A total of 101 women were excluded because of missing data on birthday of the first birth or/and the last menstrual data of the second birth. The final sample consisted of 2604 women with a mean age of 20.0 ( $\mathrm{SD}=3.8$, ranging from 12.0 to $38.0)$ years at the preceding delivery and $21.7(\mathrm{SD}=3.8$, ranged from 13.0 to 40.0 ) years at the next. Among the 2604 children, $2263(86.9 \%)$ were successfully followed at 1 year, and $2119(81.4 \%)$ were followed at 7 years.

\section{Definition of interpregnancy interval}

The interpregnancy interval was calculated as the interval between the conception date of the subsequent pregnancy and the delivery date of the preceding pregnancy [1].

\section{Maternal and perinatal characteristic}

Maternal variables used in this study included race/ethnicity (white, black, and other [mostly Puerto Rican]), prepregnancy BMI (calculated as weight in kilograms divided by height in meters squared based on prepregnancy height and weight), maternal weight gain in pregnancy (calculated from prepregnancy weight to weight at delivery), marital status (married/cohabiting vs. none), educational level ( $\geq 12$, 9-12, <9 years), socioeconomic status index (from 1 for the lowest to 5 for the highest), smoking during pregnancy ( 0 , $1-9$, and $\geq 12$ number in cigarette/day), maternal diabetes (defined as gestational and/or pregestational diabetes, yes vs. no), and maternal chronic hypertension (defined as gestational and/or pregestational hypertension, yes vs. no).

Perinatal variables included gender, mode of labor onset (spontaneous, induced, and caesarean), birthweight, genderand race-specific birthweight for gestational age group (small for gestational age (SGA, <10th percentile), appropriate for gestational age (AGA, 10th-90th percentile), and large for gestational age [LGA], $\geq 90$ th), Apgar scores at 1 and $5 \mathrm{~min}$ $(0-4,5-7$, and 8-10), and feeding pattern (exclusively formula fed, exclusively breastfed, and mixed fed).

Among all these maternal and perinatal variables, maternal height and weight, blood pressure were measured in the hospital by clinical staff as part of the routine clinical care. Child height, weight, and blood pressure were measured by trained research staff at the follow-up visit. Information collected by questionnaire was collected throug in-person interview by trained research staff on a standard maternal questionnaire.

\section{Assessment of postnatal growth, BMI and blood pressure}

Detailed physical examinations, including length/height, weight and blood pressure measures, were performed at each visit following a standard procedure until 7 years old [20]. The weight at birth and at 1 year old was standardized as age- and gender-specific $\mathrm{z}$ sores based on the World Health Organization's growth chart [21]. Weight gain in the first year of life was defined as the change in weight-for-age $\mathrm{z}$ scores between the birth and the end of the first year, and then categorized into 2 groups: rapid weight gain (change in $z$ score, $>0.67$ ) and nonrapid weight gain (change in $z$ score, $\leq 0.67$ ) [22]. The BMI at 7 years was calculated based on weight and height (weight in kilograms divided by height in meters squared). The weight, height and BMI at 7 years were standardized as age- and gender-specific $z$ sores using the US national reference data [23]. To obtain the z-score 
Table 1 Characteristics of study participants stratified by interpregnancy interval and maternal age groups

\begin{tabular}{|c|c|c|c|c|c|c|c|c|}
\hline \multirow[t]{2}{*}{ Variables no $(\%)$} & \multicolumn{3}{|c|}{ Interpregnancy interval (months) } & \multirow[t]{2}{*}{$P$ value } & \multicolumn{3}{|c|}{ Maternal age at 2nd delivery (years) } & \multirow[t]{2}{*}{$P$ value } \\
\hline & $\begin{array}{l}<6 \\
(n=787)\end{array}$ & $\begin{array}{l}6-24 \\
(n=1545)\end{array}$ & $\begin{array}{l}\geq 24 \\
(n=272)\end{array}$ & & $\begin{array}{l}<20 \\
(n=824)\end{array}$ & $\begin{array}{l}20-30 \\
(n=1664)\end{array}$ & $\begin{array}{l}\geq 30 \\
(n=116)\end{array}$ & \\
\hline \multicolumn{9}{|l|}{ Maternal characteristics } \\
\hline $\begin{array}{l}\text { Maternal age at delivery, mean } \\
\text { (SD), yrs }\end{array}$ & $20.55(3.46)$ & $21.85(3.81)$ & $23.74(3.89)$ & $<0.001$ & $17.84(1.15)$ & $22.78(2.42)$ & $32.46(2.48)$ & $<0.001$ \\
\hline $\begin{array}{l}\text { Interpregnancy interval, mean (SD), } \\
\text { mons }\end{array}$ & $3.89(1.21)$ & $12.55(4.79)$ & $33.61(8.40)$ & $<0.001$ & $8.89(7.18)$ & $12.70(10.08)$ & $\begin{array}{l}15.73 \\
(11.18)\end{array}$ & $<0.001$ \\
\hline $\begin{array}{l}\text { Prepregnancy BMI, mean (SD), kg/ } \\
\mathrm{m}^{2}\end{array}$ & $25.98(3.90)$ & $25.82(4.12)$ & $26.91(4.00)$ & $<0.001$ & $25.79(3.67)$ & $26.00(4.18)$ & $27.15(4.72)$ & 0.007 \\
\hline $\begin{array}{l}\text { Weight gain in pregnancy, mean } \\
\text { (SD), kg }\end{array}$ & $10.21(4.96)$ & $10.41(5.36)$ & $11.17(6.04)$ & 0.042 & $10.73(5.74)$ & $10.29(5.10)$ & $10.25(5.83)$ & 0.151 \\
\hline Race, $\%$ & & & & 0.866 & & & & $<0.001$ \\
\hline White & 58.18 & 58.42 & 56.25 & & 38.20 & 66.87 & 74.14 & \\
\hline Black & 37.72 & 37.82 & 40.81 & & 59.49 & 28.80 & 19.83 & \\
\hline Other & 0.64 & 0.78 & 1.10 & & - & 0.96 & 3.45 & \\
\hline Missing & 3.45 & 2.98 & 1.84 & & 2.31 & 3.37 & 2.59 & \\
\hline Marriage, $\%$ & & & & 0.028 & & & & $<0.001$ \\
\hline Yes & 88.31 & 84.40 & 83.82 & & 74.88 & 90.14 & 94.83 & \\
\hline No & 11.69 & 15.60 & 16.18 & & 25.12 & 9.86 & 5.17 & \\
\hline Education, yrs, \% & & & & 0.555 & & & & $<0.001$ \\
\hline$\geq 12$ & 50.32 & 53.66 & 55.15 & & 17.96 & 68.81 & 70.69 & \\
\hline $9-12$ & 37.99 & 35.53 & 34.19 & & 65.29 & 22.90 & 18.97 & \\
\hline$<9$ & 11.69 & 10.81 & 10.66 & & 16.75 & 8.29 & 10.34 & \\
\hline Socioeconomic status index, $\%$ & & & & 0.001 & & & & $<.001$ \\
\hline 1 (lowest) & 5.06 & 4.29 & 3.32 & & 9.77 & 1.90 & 2.61 & \\
\hline 2 & 27.63 & 24.27 & 15.50 & & 41.29 & 16.77 & 13.04 & \\
\hline 3 & 31.52 & 29.75 & 33.58 & & 33.75 & 29.25 & 29.57 & \\
\hline 4 & 24.77 & 25.86 & 28.78 & & 12.73 & 32.44 & 24.35 & \\
\hline 5 (highest) & 10.25 & 15.44 & 18.45 & & 1.36 & 19.40 & 30.43 & \\
\hline Missing & 0.78 & 0.40 & 0.37 & & 1.11 & 0.24 & - & \\
\hline $\begin{array}{l}\text { Smoking, number in cigarette/day, } \\
\%\end{array}$ & & & & 0.379 & & & & $<0.001$ \\
\hline 0 & 52.18 & 52.31 & $153(56.25)$ & & 48.11 & 54.35 & 61.40 & \\
\hline $1-9$ & 28.33 & 25.89 & 69 (25.37) & & 34.31 & 23.01 & 22.81 & \\
\hline$\geq 10$ & 19.49 & 21.80 & $50(18.38)$ & & 17.58 & 22.64 & 15.79 & \\
\hline $\begin{array}{l}\text { Pergestational/gestational diabetes, } \\
\%\end{array}$ & & & & 0.966 & & & & 0.892 \\
\hline No & 98.35 & 98.38 & 98.16 & & 98.18 & 98.44 & 98.28 & \\
\hline Yes & 1.65 & 1.62 & 1.84 & & 1.82 & 1.56 & 1.72 & \\
\hline $\begin{array}{l}\text { Pergestational/gestational } \\
\text { hypertension, } \%\end{array}$ & & & & 0.002 & & & & \\
\hline No & 83.35 & 81.10 & 73.53 & & 80.95 & 81.25 & 77.59 & 0.623 \\
\hline Yes & 16.65 & 18.90 & 26.47 & & 19.05 & 18.75 & 22.41 & \\
\hline \multicolumn{9}{|l|}{ Neonatal characteristics } \\
\hline Gender, $\%$ & & & & 0.388 & & & & 0.388 \\
\hline Male & 52.48 & 50.32 & 53.68 & & 53.03 & 50.75 & 47.41 & \\
\hline Female & 47.52 & 49.68 & 46.32 & & 46.97 & 49.25 & 52.59 & \\
\hline Mode of labor onset, $\%$ & & & & 0.002 & & & & 0.002 \\
\hline Induced & 1.40 & 2.35 & 2.95 & & 1.58 & 2.24 & 4.31 & \\
\hline
\end{tabular}


Table 1 (continued)

\begin{tabular}{|c|c|c|c|c|c|c|c|c|}
\hline \multirow[t]{2}{*}{ Variables no (\%) } & \multicolumn{3}{|c|}{ Interpregnancy interval (months) } & \multirow[t]{2}{*}{$P$ value } & \multicolumn{3}{|c|}{ Maternal age at 2nd delivery (years) } & \multirow[t]{2}{*}{$P$ value } \\
\hline & $\begin{array}{l}<6 \\
(n=787)\end{array}$ & $\begin{array}{l}6-24 \\
(n=1545)\end{array}$ & $\begin{array}{l}\geq 24 \\
(n=272)\end{array}$ & & $\begin{array}{l}<20 \\
(n=824)\end{array}$ & $\begin{array}{l}20-30 \\
(n=1664)\end{array}$ & $\begin{array}{l}\geq 30 \\
(n=116)\end{array}$ & \\
\hline Naturally & 93.49 & 91.34 & 85.24 & & 94.53 & 90.25 & 84.48 & \\
\hline Cesarean & 4.21 & 5.54 & 9.23 & & 3.41 & 6.30 & 9.48 & \\
\hline Missing & 0.89 & 0.78 & 2.58 & & 0.49 & 1.21 & 1.72 & \\
\hline $\begin{array}{l}\text { Gestational age at birth, mean (SD), } \\
\text { wk }\end{array}$ & $38.77(3.04)$ & $38.72(2.93)$ & $38.88(2.53)$ & 0.702 & $38.12(3.45)$ & $39.07(2.60)$ & $38.67(2.52)$ & $<0.001$ \\
\hline Birthweight, mean (SD), kg & $3.17(0.53)$ & $3.22(0.50)$ & $3.20(0.48)$ & 0.085 & $3.09(0.52)$ & $3.25(0.51)$ & $3.22(0.45)$ & $<0.001$ \\
\hline Birthweight, mean (SD), z score & $-0.62(1.12)$ & $-0.52(1.07)$ & $-0.57(1.04)$ & 0.083 & $\begin{array}{l}-0.77 \\
(1.13)\end{array}$ & $-0.44(1.04)$ & $\begin{array}{l}-0.52 \\
(1.08)\end{array}$ & $<0.001$ \\
\hline Birthweight for gestational age, $\%$ & & & & 0.667 & & & & 0.321 \\
\hline SGA & 9.81 & 9.05 & 8.68 & & 10.71 & 8.59 & 7.96 & \\
\hline AGA & 80.77 & 79.54 & 80.75 & & 79.58 & 80.05 & 83.19 & \\
\hline LGA & 9.42 & 11.40 & 10.57 & & 9.71 & 11.36 & 8.85 & \\
\hline Apgar score at $5 \mathrm{~min}, \%$ & & & & 0.661 & & & & 0.036 \\
\hline $0-4$ & 3.30 & 3.41 & 4.20 & & 2.55 & 3.90 & 3.54 & \\
\hline $5-7$ & 3.70 & 2.68 & 3.44 & & 4.46 & 2.47 & 1.77 & \\
\hline $8-10$ & 93.00 & 93.91 & 92.37 & & 92.98 & 93.63 & 94.69 & \\
\hline \multicolumn{9}{|l|}{ Growth characteristics } \\
\hline Feeding pattern, $\%$ & & & & 0.214 & & & & $<0.001$ \\
\hline Exclusively breastfed & 7.93 & 10.86 & 9.51 & & 5.20 & 11.48 & 19.30 & \\
\hline Exclusively formular & 82.57 & 80.07 & 82.89 & & 89.60 & 77.90 & 66.67 & \\
\hline Mixed & 7.80 & 8.87 & 7.60 & & 4.58 & 9.94 & 14.04 & \\
\hline Missing & 1.69 & 0.20 & - & & 0.62 & 0.68 & - & \\
\hline $\begin{array}{l}\text { Weight gain in the } 1 \text { st } y r \text {, mean } \\
\text { (SD), } \mathrm{kg}\end{array}$ & $6.57(1.18)$ & $6.61(1.16)$ & $6.71(1.22)$ & 0.296 & $6.49(1.19)$ & $6.43(1.16)$ & $6.89(1.24)$ & $<0.001$ \\
\hline $\begin{array}{l}\text { Weight gain in the } 1 \text { st } y r \text {, mean } \\
\text { (SD), z score }\end{array}$ & $0.41(1.28)$ & $0.42(1.22)$ & $0.49(1.33)$ & 0.717 & $0.44(1.29)$ & $0.40(1.23)$ & $0.71(1.31)$ & 0.214 \\
\hline $\begin{array}{l}\text { Rapid weight gain in the first year, } \\
\%\end{array}$ & & & & 0.869 & & & & 0.147 \\
\hline Nonrapid & 66.84 & 66.41 & 65.07 & & $65.78)$ & 67.25 & 58.62 & \\
\hline Rapid & 33.16 & 33.59 & 34.93 & & 34.22 & 32.75 & 41.38 & \\
\hline \multicolumn{9}{|l|}{ Characteristics at 7 years } \\
\hline Weight, mean (SD), cm & $23.72(3.93)$ & $23.96(4.26)$ & $24.38(4.48)$ & 0.124 & $23.66(4.02)$ & $23.95(4.20)$ & $25.55(5.37)$ & $<0.001$ \\
\hline Weight, mean (SD), z score & $0.05(0.94)$ & $0.09(1.01)$ & $0.19(1.01)$ & 0.214 & $0.03(0.96)$ & $0.10(0.99)$ & $0.43(1.05)$ & 0.001 \\
\hline Height, mean (SD), $\mathrm{kg} / \mathrm{m}^{2}$ & $\begin{array}{l}121.26 \\
(5.84)\end{array}$ & $121.24(6.01)$ & $\begin{array}{l}121.27 \\
(5.92)\end{array}$ & 0.995 & $\begin{array}{l}121.34 \\
(5.95)\end{array}$ & $121.16(5.97)$ & $\begin{array}{l}121.84 \\
(6.56)\end{array}$ & 0.502 \\
\hline Height, mean (SD), z score & $-0.09(1.06)$ & $-0.10(1.10)$ & $-0.10(1.08)$ & 0.997 & $\begin{array}{l}-0.07 \\
(1.09)\end{array}$ & $-0.10(1.09)$ & $0.02(1.18)$ & 0.508 \\
\hline BMI, mean (SD), $\mathrm{kg} / \mathrm{m}^{2}$ & $16.06(1.72)$ & $16.18(1.92)$ & $16.52(2.09)$ & 0.007 & $15.97(1.70)$ & $16.23(1.97)$ & $17.08(2.32)$ & $<0.001$ \\
\hline BMI, mean (SD), z score & $0.17(0.89)$ & $0.21(0.94)$ & $0.38(0.91)$ & 0.013 & $0.12(0.90)$ & $0.24(0.95)$ & $0.62(0.95)$ & $<0.001$ \\
\hline $\mathrm{SBP}$, mean (SD), mmHg & $\begin{array}{l}101.30 \\
(10.38)\end{array}$ & $101.60(9.98)$ & $\begin{array}{l}102.20 \\
(10.37)\end{array}$ & 0.521 & $\begin{array}{l}100.70 \\
(9.88)\end{array}$ & $\begin{array}{l}101.77 \\
(10.27)\end{array}$ & $\begin{array}{l}105.07 \\
(9.94)\end{array}$ & $<0.001$ \\
\hline $\mathrm{DBP}$, mean $(\mathrm{SD}), \mathrm{mmHg}$ & $60.02(9.75)$ & $60.62(10.04)$ & $62.58(9.32)$ & 0.005 & $\begin{array}{l}59.78 \\
(10.18)\end{array}$ & $60.91(9.70)$ & $\begin{array}{l}62.97 \\
(10.98)\end{array}$ & 0.004 \\
\hline
\end{tabular}

$S G A$ small for gestational age, $A G A$ appropriate for gestational age, $L G A$ large for gestational age, $B M I$ body mass index (calculated as weight in kilograms divided by height in meters square) 
for a given measurement (x), the following equation was used: $\mathrm{Z}=\left\{\left[(\mathrm{x} / \mathrm{M})^{* * \mathrm{~L}]}-1\right\} / \mathrm{LS}(\mathrm{L} \neq 0)\right.$ or $\mathrm{Z}=\ln (\mathrm{x} / \mathrm{M}) / \mathrm{S}(\mathrm{L}$ $=0)(\mathrm{M}$, median; $\mathrm{S}$, the generalized coefficient of variation, and L, the power in the Box-Cox transformation) [23].

\section{Statistical analyses}

Summary statistics and distributional plots were checked for all variables. One-way ANOVA and Chi-square test were used to compare differences between groups where appropriate. Locally weighted nonparametric smoothing plots (SAS LOESS; SAS Institute, Cary, NC) were used to graphically examine the relationship between interpregnancy interval and maternal age at delivery. Similarly, LOESS was used to model the relationship of interpregnancy interval with childhood BMI z scores, SBP and DBP. BMI z scores, SBP and DBP were grouped into three maternal age groups for each week of interpregnancy interval. Stratified by maternal age groups ( $<20,20-30$, and $\geq 30$ years), BMI z scores, SBP and DBP were plotted against interpregnancy interval.

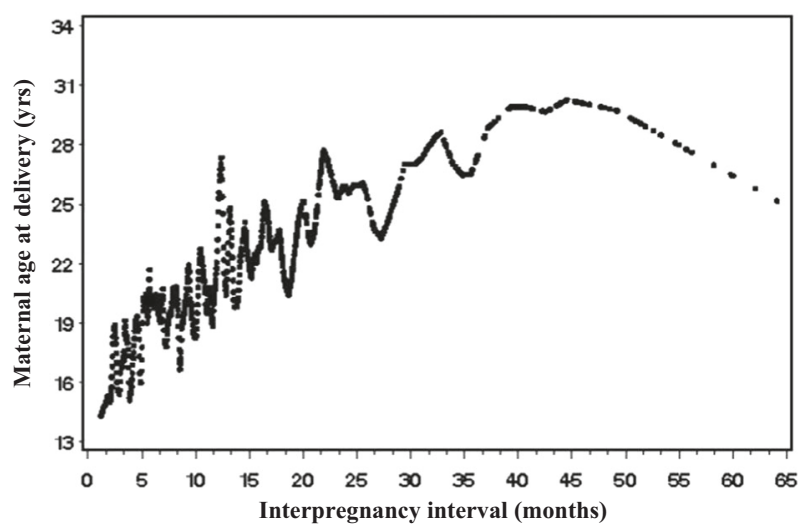

Fig. 1 Smoothed plots of maternal age at delivery with interpregnancy interval
Generalized linear regression models were further applied to estimate the crude and adjusted associations of interpregnancy interval with BMI $\mathrm{z}$ scores, SBP and DBP. Adjustments were made following a four-step procedure. Model 1 was adjusted for all maternal and perinatal variables (as shown in Table 1). In model $2^{\mathrm{a}}$, postnatal variables such as feeding pattern and rapid weight gain in the first year of life, were further controlled. Previous studies have demonstrated that BMI and height are associated with blood pressure $[24,25]$. Therefore, BMI z score and height $z$ score at 7 years were additionally adjusted in model $2^{\mathrm{b}}$. Subsequently, maternal age at delivery was added to determine the associations of maternal age at delivery and offspring's BMI z score, SBP and DBP, and, meanwhile, to test whether maternal age mediates the associations. Finally, the interaction between interpregnancy interval and maternal age was simultaneously adjusted to examine if maternal age was an effect modifier in the association of interpregnancy interval with BMI z score, SBP and DBP.

Finally, we undertook a sensitivity analysis in which we restricted our analyses to those children with appropriate birthweight for gestational age $(n=1997)$ based on the recent evidence that birthweight was directly associated with childhood blood pressure [26].

\section{Results \\ Characteristics of participants}

A total of 2604 woman-child pairs were included in this study. The mean interpregnancy interval was $12.1(\mathrm{SD}=9.5)$ months. The mean maternal age was $20.0(\mathrm{SD}=3.8)$ years at the preceding delivery and $21.7(\mathrm{SD}=3.8)$ years at the index pregnancy. The corresponding gestational age was 39.1 (SD $=2.8$ ) weeks and $38.8(\mathrm{SD}=2.9)$ weeks, respectively. There was a trend of increased maternal age at the index pregnancy
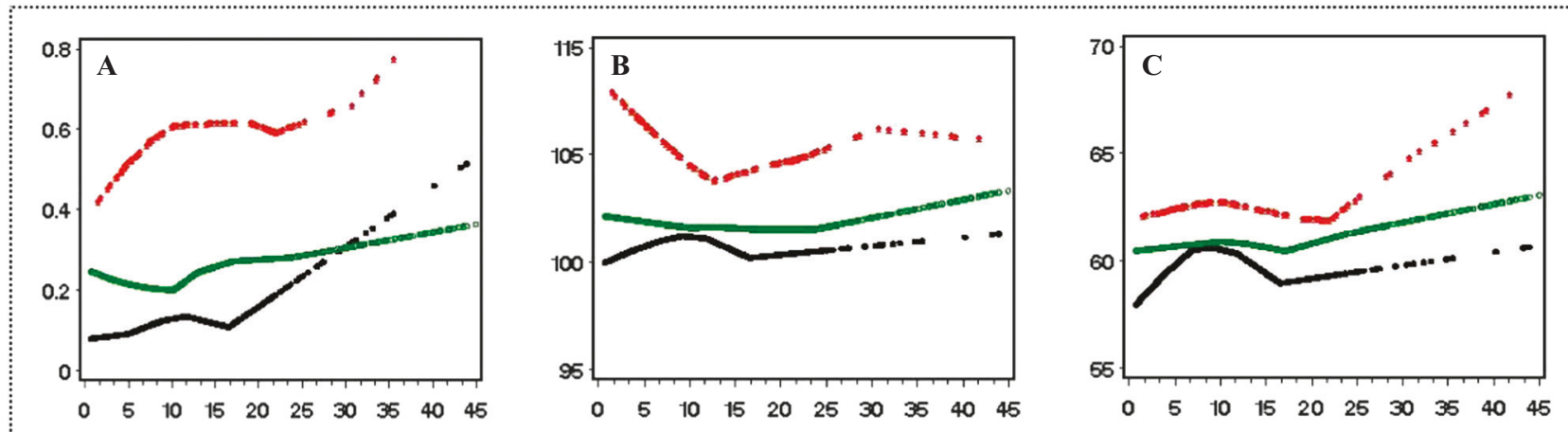

Interpregnancy interval (months)

Fig. 2 Smoothed plots of BMI z score, SBP and DBP with interpregnancy interval, stratified by the maternal age groups a BMI z score, $\mathbf{b}$ SBP, and c DBP Maternal age groups: black for $<20$ yrs, green for $20-30 \mathrm{yrs}$, red for $\geq 30 \mathrm{yrs}$ 
Table 2 Associations of interpregnancy interval (analyzed as continuous variable), maternal age at delivery (analyzed as continuous variable) with BMI z score, SBP, and DBP at 7 years (analyzed as continuous variable)

\begin{tabular}{|c|c|c|c|c|}
\hline & \multicolumn{2}{|c|}{ Interpregnancy Interval (months) } & \multicolumn{2}{|l|}{ Maternal Age (years) } \\
\hline & $\beta(95 \% \mathrm{CI})$ & $P$ value & $\beta(95 \% \mathrm{CI})$ & $P$ value \\
\hline \multicolumn{5}{|c|}{ BMI at $7 \mathrm{yrs}, \mathrm{z}$ score } \\
\hline Crude & $0.007(0.003,0.011)$ & $<0.001$ & & \\
\hline \multicolumn{5}{|c|}{ Adjusted } \\
\hline 1 & $0.004(0.001,0.009)$ & 0.048 & & \\
\hline $2^{\mathrm{a}}$ & $0.003(-0.001,0.007)$ & 0.134 & & \\
\hline $3^{\mathrm{a}}$ & $0.002(-0.003,0.006)$ & 0.428 & $0.014(0.001,0.027)$ & 0.036 \\
\hline \multicolumn{5}{|c|}{$\mathrm{SBP}$ at $7 \mathrm{yrs}, \mathrm{mmHg}$} \\
\hline Crude & $0.020(-0.026,0.065)$ & 0.394 & & \\
\hline \multicolumn{5}{|c|}{ Adjusted } \\
\hline 1 & $0.018(0.032,0.068)$ & 0.490 & & \\
\hline $2^{\mathrm{a}}$ & $0.006(-0.044,0.055)$ & 0.824 & & \\
\hline $2^{b}$ & $0.003(-0.046,0.051)$ & 0.920 & & \\
\hline $3^{\mathrm{b}}$ & $-0.010(-0.061,0.040)$ & 0.693 & $0.123(-0.026,0.271)$ & 0.105 \\
\hline \multicolumn{5}{|c|}{$\mathrm{DBP}$ at $7 \mathrm{yrs}, \mathrm{mmHg}$} \\
\hline Crude & $0.057(0.013,0.101)$ & 0.012 & & \\
\hline \multicolumn{5}{|c|}{ Adjusted } \\
\hline 1 & $0.058(0.010,0.106)$ & 0.021 & & \\
\hline $2^{\mathrm{a}}$ & $0.054(0.005,0.104)$ & 0.031 & & \\
\hline $2^{\mathrm{b}}$ & $0.053(0.004,0.102)$ & 0.033 & & \\
\hline $3^{b^{*}}$ & $0.051(0.001,0.103)$ & 0.049 & $0.017(-0.133,0.167)$ & 0.824 \\
\hline
\end{tabular}

Model 1: adjusted for infant gender, maternal race, maternal marriage status, SES, mother's educational level, smoking status, pregestational/ gestational diabetes, pergestational/gestational hypertension, gestational age, prepregnancy BMI, weight gain in pregnancy, mode of labor onset, birthweight for gestational age, Apgar score at 5 min

Model $2^{\mathrm{a}}$ : model 1 adjustments, as well as feeding pattern and rapid weight gain in the first year of life

Model $2^{\mathrm{b}}$ : model $2^{\mathrm{a}}$ adjustments, as well as BMI $\mathrm{z}$ score and height $\mathrm{z}$ score at 7 years

Model $3^{\mathrm{a}}$ : model $2^{\mathrm{a}}$ adjustments, as well as maternal age at delivery

Model $3^{\mathrm{b}}$ : model $2^{\mathrm{b}}$ adjustments, as well as maternal age at delivery

${ }^{*} P<0.05$ for interaction interpregnancy interval*maternal age

with lengthened interpregnancy interval $(p<0.001)$ (Fig. 1). Table 1 shows the detailed information of sample characteristics for different interpregnancy interval/maternal age groups. No differences were observed in maternal race and maternal education among the three interpregnancy interval groups. However, marital status and socioeconomic status index differed among the three groups, where it was shown that married women tended to have shorter interpregnancy interval and maternal higher socioeconomic status index correlated to longer interpregnancy interval.

\section{The associations of interpregnancy interval, maternal age, and offspring's BMI and BP}

Figure 2 shows the smoothing distributions of BMI $z$ score, systolic BP and diastolic BP with increasing interpregnancy interval, stratified by different maternal age groups. BMI $z$ score and diastolic BP were positively associated with inerpregnancy interval, and the associations were independent of maternal age group.

Tables 2 and 3 present the crude and the adjusted associations of interpregnancy interval with offspring's BMI and $\mathrm{BP}$ at 7 years, where interpregnancy interval and maternal age were analyzed as continuous (Table 2) and categorical (Table 3) variables, respectively. In crude models, we found independent associations of interpregnancy interval with offspring's BMI z score and diastolic BP at 7 years, and this was consistent when interpregnancy interval was analyzed as either a continuous or categorical variable. The association between interpregnancy interval and diastolic BP remained statistically significant through three-step adjustment for potential confounders. From model 1 to model $2^{\mathrm{b}}$, the effect size changed little. Further adjustment for maternal age at delivery in the model (model $3^{\mathrm{b}}$ ) did not change the association between interpregnancy interval and offspring's diastolic BP at 7 years, indicating no mediating 
Table 3 Associations of interpregnancy interval (analyzed as categorical variable), maternal age at delivery (analyzed as categorical variable) and BMI, SBP, and DBP at 7 years (analyzed as continuous variable)

\begin{tabular}{|c|c|c|c|c|c|c|c|c|}
\hline & \multicolumn{4}{|c|}{ Interpregnancy interval (months) } & \multicolumn{4}{|c|}{ Maternal age at delivery (years) } \\
\hline & \multicolumn{2}{|l|}{$6-24$ vs. $<6$} & \multicolumn{2}{|l|}{$\geq 24$ vs. $<6$} & \multicolumn{2}{|l|}{$20-30$ vs. $<20$} & \multicolumn{2}{|l|}{$\geq 30$ vs. $<20$} \\
\hline & $\beta(95 \% \mathrm{CI})$ & $P$ value & $\beta(95 \% \mathrm{CI})$ & $P$ value & $\beta(95 \% \mathrm{CI})$ & $P$ value & $\beta(95 \% \mathrm{CI})$ & $P$ value \\
\hline \multicolumn{9}{|c|}{ BMI at $7 \mathrm{yrs}, \mathrm{z}$ score } \\
\hline Crude & $0.040(-0.047,0.128)$ & 0.369 & $0.212(0.070,0.353)$ & 0.003 & & & & \\
\hline \multicolumn{9}{|c|}{ Adjusted } \\
\hline 1 & $0.017(-0.076,0.109)$ & 0.725 & $0.134(-0.016,0.283)$ & 0.080 & & & & \\
\hline $2^{\mathrm{a}}$ & $0.003(-0.088,0.094)$ & 0.946 & $0.102(-0.045,0.244)$ & 0.175 & & & & \\
\hline $3^{\mathrm{a}}$ & $-0.003(-0.095,0.089)$ & 0.948 & $0.084(-0.066,0.234)$ & 0.274 & $-0.002(-0.109,0.104)$ & 0.965 & $0.287(0.065,0.510)$ & 0.012 \\
\hline \multicolumn{9}{|c|}{$\mathrm{SBP}$ at $7 \mathrm{yrs}, \mathrm{mmHg}$} \\
\hline Crude & $0.300(-0.668,1.268)$ & 0.544 & $0.903(-0.661,2.467)$ & 0.258 & & & & \\
\hline \multicolumn{9}{|c|}{ Adjusted } \\
\hline 1 & $0.447(-0.633,1.526)$ & 0.417 & $0.346(-1.404,2.095)$ & 0.699 & & & & \\
\hline $2^{\mathrm{a}}$ & $0.348(-0.736,1.431)$ & 0.529 & $0.085(-1.663,1.833)$ & 0.924 & & & & \\
\hline $2^{\mathrm{b}}$ & $0.306(-0.744,1.356)$ & 0.568 & $-0.074(-1.768,1.620)$ & 0.932 & & & & \\
\hline $3^{\mathrm{b}}$ & $0.235(-0.826,1.295)$ & 0.665 & $-0.260(-1.991,1.471)$ & 0.769 & $0.360(-1.771,0.742)$ & 0.422 & $\begin{array}{l}0.103(-2.526 \\
2.732)\end{array}$ & 0.939 \\
\hline \multicolumn{9}{|c|}{$\mathrm{DBP}$ at $7 \mathrm{yrs}, \mathrm{mmHg}$} \\
\hline Crude & $0.579(-0.366,1.525)$ & 0.230 & $2.553(1.025,4.079)$ & 0.001 & & & & \\
\hline \multicolumn{9}{|c|}{ Adjusted } \\
\hline 1 & $0.046(-1.019,1.111)$ & 0.932 & $1.837(0.111,3.562)$ & 0.037 & & & & \\
\hline $2^{\mathrm{a}}$ & $0.004(-1.071,1.080)$ & 0.994 & $1.772(0.039,3.506)$ & 0.045 & & & & \\
\hline $2^{\mathrm{b}}$ & $-0.056(-1.124,1.012)$ & 0.919 & $1.692(0.003,3.414)$ & 0.046 & & & & \\
\hline $3^{\mathrm{b}^{*}}$ & $-0.009(-1.087,1.069)$ & 0.987 & $1.794(0.034,3.554)$ & 0.046 & $-0.515(-1.771,0.742)$ & 0.422 & $\begin{array}{l}0.103(-2.526 \\
2.732)\end{array}$ & 0.939 \\
\hline
\end{tabular}

Model 1: adjusted for infant gender, maternal race, maternal marriage status, SES, mother's educational level, smoking status, pregestational/ gestational diabetes, gestational age, prepregnancy BMI, weight gain in pregnancy, mode of labor onset, birthweight for gestational age, Apgar score at $5 \mathrm{~min}$

Model $2^{\text {a }}$ : model 1 adjustments, as well as birthweight, feeding pattern, rapid weight gain in the first year of life

Model $2^{\mathrm{b}}$ : model $2^{\mathrm{a}}$ adjustments, as well as BMI z score and height $\mathrm{z}$ score at 7 years

Model $3^{\mathrm{a}}$ : model $2^{\mathrm{a}}$ adjustments, as well as maternal age at delivery

Model $3^{\mathrm{b}}$ : model $2^{\mathrm{b}}$ adjustments, as well as maternal age at delivery

${ }^{*} P<0.05$ for interaction interpregnancy interval $\times$ maternal age

effect of maternal age on the association. Finally, when examining the interaction effect between interpregnancy interval and maternal age at delivey in the associations of interpregnancy interval with offspring's BMI and BP at 7 years, we found a significant interaction term in the association with diastolic BP, and this was valid when interpregnancy interval was analyzed as both a continuous (Table 2, $p$ for interaction $=0.019$ ) and categorical variable (Table 3, $p$ for interaction $=0.049$ ). The crude and the adjusted associations of maternal age with offspring's BMI and blood pressure at 7 years were shown in Online Tables 1 and 2. After adjusting for confounders, the significant association between maternal age and BMI z score was established and the association was independent of interpregnancy interval.
We regrouped our study sample into 9 groups in Fig. 3 . A general pattern was found that offspring whose mother had longer interpregnancy interval and older maternal age had higher BMI z score and DBP in particular. When the above analyses were restricted to children with appropriate birthweight for gestational age $(n=1997)$, the results remained essentially the same (not shown).

\section{Discussion}

Our prospective cohort study shows that interpregnancy interval was independently positively associated with offspring's diastolic BP, and maternal age was independently positively associated with offspring's BMI z score at age 7 


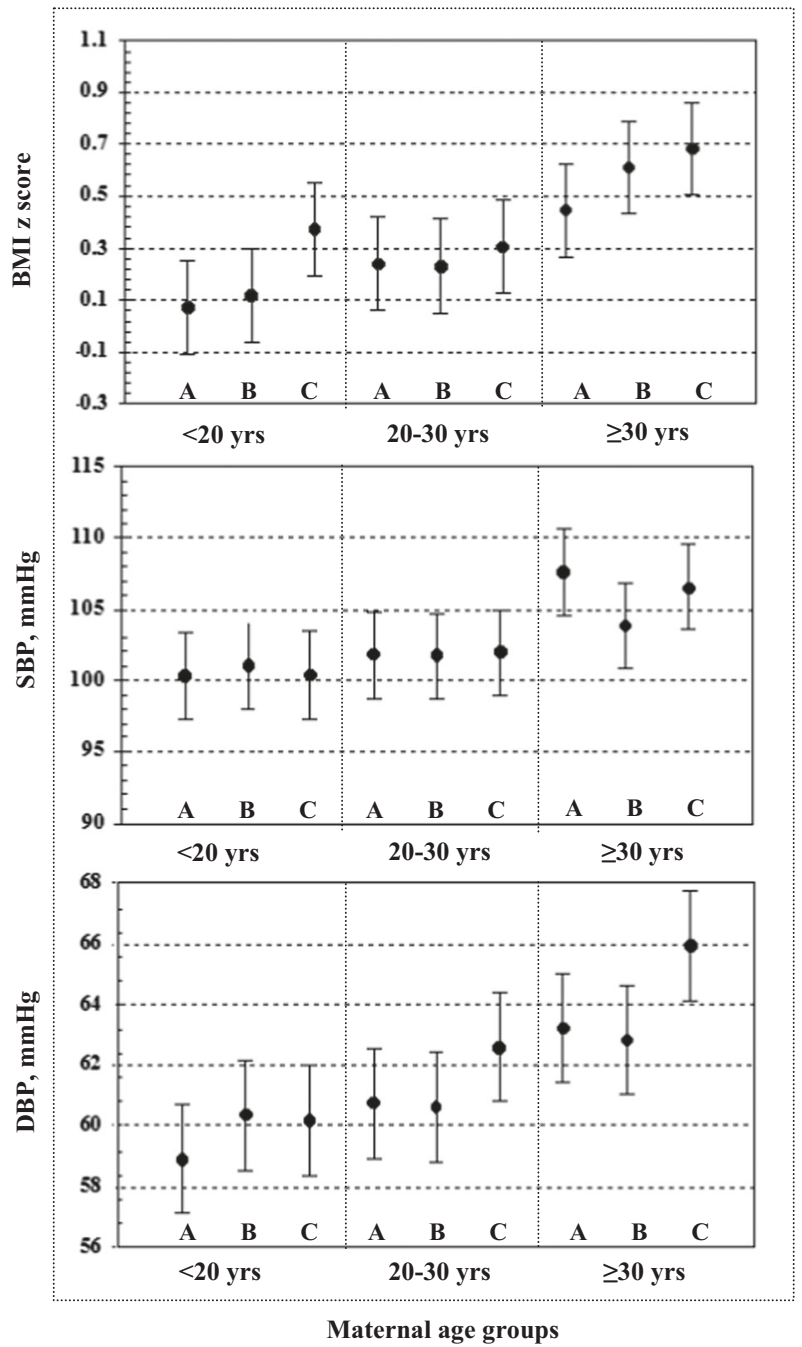

Fig. 3 Interaction effect of interpregnancy interval and maternal age at delivery on BMI at 7 years a interpregnancy interval $<6$ monthss, b 6-24 months, $\mathbf{c} \geq 24$ months

yrs. Increasing maternal age could amplify the individual association between interpregnancy interval and offspring's diastolic BP. Interpregnancy interval $\geq 24$ months, especially for women of advanced age, had long-term negative impacts on childhood health. This is the first study to report the long-term impacts of interpregnancy interval on childhood growth and development.

Growing evidence suggests that interpregnancy interval is associated with fetal growth and perinatal outcome; however, the results are inconsistent [2-7, 17-19]. Traditionally, short interpregnancy interval, defined as $<6$ months between the birth of one child and the conception of the next, is regarded as a risk factor for fetal and perinatal health [2-7]. The current WHO recommendation for ideal interpregnancy interval is no $<24$ months after a live birth [16]. However, a recent study re-evaluated and questioned the causal effect of interpregnancy interval on adverse birth outcomes after thorough adjustment for persistent maternal factor by using a within mother analysis [17]. The same study also pointed to the importance of taking maternal factors into account when evaluating the role of interpregnancy interval in maternal health and offspring's growth and development [17]. Similarly, a few studies have questioned the WHO recommendation and suggested shortening the recommendation for ideal interpregnancy interval especially for women of advanced age $[18,19]$. Our study did not identify the negative effect of short interpregnancy interval, whereas we found that lengthened interpregnancy interval was associated with offspring's higher diastolic BP at 7 years, and the coexisting with older maternal age aggravated the effect. Based on our findings, an interpregnancy interval $<24$ months, especially for women older than 30 years is optimal for child health outcomes.

It is becoming a general recognition that perinatal events are involved in the fetal programming and developmental origins of health and disease $[10,11]$. Epidemiological studies have identified a link between perinatal markers, such as maternal pre-pregnancy BMI, duration of pregnancy, birthweight, feeding pattern, and early postnatal growth, with offspring's blood pressure and metabolic profile later in life [26-29]. After adjusting for all these factors and other possible confounders, we established a positive association between interpregnancy interval and offspring's diastolic BP at 7 years, and the association was aggravated with increasing maternal age. Two previous studies found the relationship between short interpregnancy interval $(<2-3$ yrs) and a lower maternal mean arterial blood pressure in the following pregnancy $[20,30]$. The possible mechanism is the cardiovascular adaptation achieved during one pregnancy, which may facilitate vascular compliance in the next pregnancy; and the established cardiovascular adaptation only maintain within relatively short interpregnancy interval [31]. We speculate that the cardiovascular adaptation may play a long-term role in offspring's growth and metabolism since previous studies indicated that the cardiovascular adaptation may provide a more favorable environment for placental development, placental function, and fetal nutrition in the next pregnancy $[32,33]$.

In addition, it was reported that the advanced maternal age was associated with restricted uterine spiral vasculature volume [32]. Therefore, it is possible that advanced maternal age may adversely influence the development and duration of cardiovascular adaptation. This may explain, in part, why our study observed an aggravated effect of advanced maternal age on the positive association between interpregnancy interval and offspring's blood pressure. In contrary to the association of interpregnancy interval with offspring's childhood DBP, we did not establish the relationship between interpregnancy interval and offspring's $\mathrm{SBP}$, even with increasing maternal age. In view of the fact 
that the age of offspring in our study was still young, we considered that the relationship might arise at a later age. A recent study indicated that diastolic BP, compared to systolic BP, could be more sensitive to unfavorable environment and events in children and adolescent [34].

The main limitation of this study lies in the age of the data. Women in this study were generally young, and the total duration between the first recruitment and the last birth was only 6 years in CPP, which prevented us from exploring the effects of even longer interpregnancy intervals (longer than 3 yrs) and older maternal age (older than $35 \mathrm{yrs}$ old). However, it is reasonable to deduce that the adverse effects would be aggravated. In addition, women are now entering pregnancy with a higher BMI, and this may increse the risk of pregnancy complications and the health risk of foetus [35]. The changes should also be taken into accout when understanding the findings.

\section{Conclusion}

Our study emphasizes the importance of optimal interpregnancy interval and maternal age in offspring's health. Based on our findings, we would suggest lowering the recommendation of interpregnancy interval to no longer than 24 months and even shorter for women older than 30 years of age. As the age of women having their first child is increasing worldwide, our finding could be particularly relevant.

\section{Summary}

\section{What is known about topic}

- Short interpregnancy interval, less than 6 months, with adverse birth outcomes.

- Maternal age is an important influential factor for both fetal and postnatal growth and development.

\section{What this study adds}

- Lengthened interpregnancy interval is a risk factor for higher childhood diastolic blood pressure.

- Increasing maternal age is independently associated with offspring's higher BMI.

- There is an interaction between interpregnancy interval and maternal age in the development of childhood diastolic BP, in which increasing maternal age could aggravate the effects of prolonged interpregnancy interval.

- We would suggest lowering the current WHO recommendation for ideal interpregnancy interval to less than 24 months and even shorter for women of advanced age.
Acknowledgements This study was supported by the National Natural Science Foundation of China (81673183, 81072314), the Science and Technology Funds from Pudong New Area, Shanghai (PKJ2017Y01), the Research Funds from Shanghai Jiaotong University School of Medicine (20170509-1), and Shanghai Municipal Health and Family Planning Commission (15GWZK0401).

\section{Compliance with ethical standards}

Conflict interest The authors declare that they have no conflict of interest.

Open Access This article is licensed under a Creative Commons Attribution-NonCommercial-NoDerivatives 4.0 International License, which permits any non-commercial use, sharing, distribution and reproduction in any medium or format, as long as you give appropriate credit to the original author(s) and the source, and provide a link to the Creative Commons license. You do not have permission under this license to share adapted material derived from this article or parts of it. The images or other third party material in this article are included in the article's Creative Commons license, unless indicated otherwise in a credit line to the material. If material is not included in the article's Creative Commons license and your intended use is not permitted by statutory regulation or exceeds the permitted use, you will need to obtain permission directly from the copyright holder. To view a copy of this license, http://creativecommons.org/licenses/by-nc-nd/4.0/.

\section{References}

1. Shachar BZ, Lyell DJ. Interpregnancy interval and obstetrical complications. Obstet Gynecol Surv. 2012;67:584-96.

2. Grisaru-Granovsky S, Gordon ES, Haklai Z, Samueloff A, Schimmel MM. Effect of interpregnancy interval on adverse perinatal outcomes-a national study. Contraception. 2009;80:512-8.

3. Adam I, Ismail MH, Nasr AM, Prins MH, Smits LJ. Low birth weight, preterm birth and short interpregnancy interval in Sudan. J Matern Fetal Neonatal Med. 2009;22:1068-71.

4. Conde-Agudelo A, Rosas-Bermudez A, Kafury-Goeta AC. Birth spacing and risk of adverse perinatal outcomes: a meta-analysis. J Am Med Assoc. 2006;295:1809-23.

5. Chen I, Jhangri GS, Chandra S. Relationship between interpregnancy interval and congenital anomalies. Am J Obstet Gynecol. 2014;210(564):e1-8.

6. Kwon S, Lazo-Escalante M, Villaran MV, Li CI. Relationship between interpregnancy interval and birth defects in Washington State. J Perinatol. 2012;32:45-50.

7. Hussaini KS, Ritenour D, Coonrod DV. Interpregnancy intervals and the risk for infant mortality: a case control study of Arizona infants 2003-2007. Matern Child Health J. 2013;17:646-53.

8. Miller JE. Birth intervals and perinatal health: an investigation of three hypotheses. Fam Plann Perspect. 1991;23:62-70.

9. Nilsen RM1, Mastroiacovo P, Gunnes N, Alsaker ER, BjorkeMonsen AL, Eussen SJ, et al. Folic acid supplementation and interpregnancy interval. Paediatr Perinat Epidemiol. 2014;28:270-4.

10. Saffery R, Novakovic B. Epigenetics as the mediator of fetal programming of adult onset disease: what is the evidence? Acta Obstet Gynecol Scand. 2014;93:1090-8.

11. Gluckman PD, Hanson MA, Cooper C, Thornburg KL. Effect of in utero and early-life conditions on adult health and disease. $\mathrm{N}$ Engl J Med. 2008;359:61-73.

12. de Weger FJ, Hukkelhoven CW, Serroyen J, te Velde ER, Smits LJ. Advanced maternal age, short interpregnancy interval, 
and perinatal outcome. Am J Obstet Gynecol. 2011;204(421): e1-9.

13. Benzies KM. Advanced maternal age: are decisions about the timing of child-bearing a failure to understand the risks? CMAJ. 2008;178:183-4.

14. Laopaiboon M, Lumbiganon $\mathrm{P}$, Intarut N, Mori R, Ganchimeg T, Vogel JP, et al. Advanced maternal age and pregnancy outcomes: a multicountry assessment. WHO Multicountry Survey on Maternal Newborn Health Research Network. BJOG. 2014;121:49-56.

15. Varela-Silva MI, Azcorra H, Dickinson F, Bogin B, Frisancho AR. Influence of maternal stature, pregnancy age, and infant birth weight on growth during childhood in Yucatan, Mexico: a test of the intergenerational effects hypothesis. Am J Hum Biol. 2009;21:657-63.

16. World Health Organization. Report of a WHO Technical Consultation on Birth Spacing. Geneva, Switzerland: WHO; 2006.

17. Ball SJ, Pereira G, Jacoby P, de Klerk N, Stanley FJ. Reevaluation of link between interpregnancy interval and adverse birth outcomes: retrospective cohort study matching two intervals per mother. Br Med J. 2014;349:g4333.

18. Zhu BP, Rolfs RT, Nangle BE, Horan JM. Effect of the interval between pregnancies on perinatal outcomes. N Engl J Med. 1999;340:589-94.

19. Conde-Agudelo A, Belizan JM, Norton MH, Rosas-Bermudez A. Effect of the interpregnancy interval on perinatal outcomes in Latin America. Obstet Gynecol. 2005;106:359-66.

20. Klebanoff MA. The Collaborative Perinatal Project: a 50-year retrospective. Paediatr Perinat Epidemiol. 2009;23:2-8.

21. World Health Organization. Child growth standards. http://www. who.int/childgrowth/standards/bmi_for_age/en/index.html. Accessed November 26, 2013.

22. Leunissen RW, Kerkhof GF, Stijnen T, Hokken-Koelega A. Timing and tempo of first-year rapid growth in relation to cardiovascular and metabolic risk profile in early adulthood. J Am Med Assoc. 2009;301:2234-42.

23. Centers for Disease Control and Prevention. Growth charts. http:// www.cdc.gov/growthcharts/clinical_charts.htm. Updated August 4, 2009. Accessed November 26, 2013

24. Howe LD, Chaturvedi N, Lawlor DA, Ferreira DL, Fraser A, Davey Smith G, et al. Rapid increases in infant adiposity and overweight/obesity in childhood are associated with higher central and brachial blood pressure in early adulthood. J Hypertens. 2014;32:1789-96.

25. Regnault N, Kleinman KP, Rifas-Shiman SL, Langenberg C, Lipshultz SE, Gillman MW. Components of height and blood pressure in childhood. Int J Epidemiol. 2014;43:149-59.

26. Steinthorsdottir SD, Eliasdottir SB, Indridason OS, Palsson R, Edvardsson VO. The relationship between birth weight and blood pressure in childhood: a population-based study. Am J Hypertens. 2013;26:76-82.

27. Oostvogels AJ1, Stronks K, Roseboom TJ, van der Post JA, van Eijsden M, Vrijkotte TG. Maternal prepregnancy BMI, offspring's early postnatal growth, and metabolic profile at age 5-6 years: the ABCD Study. J Clin Endocrinol Metab. 2014;99:3845-54.

28. Lawlor DA, Najman JM, Sterne J, Williams GM, Ebrahim S, Davey Smith G. Associations of parental, birth, and early life characteristics with systolic blood pressure at 5 years of age: findings from the Mater-University study of pregnancy and its outcomes. Circulation. 2014;110:2417-23.

29. Mikolajczyk RT1, Zhang J, Ford J, Grewal J. Effects of interpregnancy interval on blood pressure in consecutive pregnancies. Am J Epidemiol. 2008;168:422-6.

30. Bernstein IM, Thibault A, Mongeon JA, Badger GJ. The influence of pregnancy on arterial compliance. Obstet Gynecol. 2005; 105:621-5.

31. Morris EA, Hale SA, Badger GJ, Magness RR, Bernstein IM. Pregnancy induces persistent changes in vascular compliance in primiparous women. Am J Obstet Gynecol. 2015;212:633.

32. Zalud I, Shaha S. Three-dimensional sonography of the placental and uterine spiralvasculature: influence of maternal age and parity. J Clin Ultrasound. 2008;36:391-6.

33. Clapp JF 3rd, Capeless E. Cardiovascular function before, during, and after the first and subsequent pregnancies. Am J Cardiol. 1997;80:1469-73.

34. Flynn J1, Zhang Y, Solar-Yohay S, Shi V. Clinical and demographic characteristics of children with hypertension. Hypertension. 2012;60:1047-54.

35. Institute of Medicine, National Research Council. Weight gain during pregnancy: reexamining the guidelines. Washington DC: The National Academic Press; 2009. 